\title{
TU/e EmonOWEN

\section{Magnetoelastic buckling of two nearby ferromagnetic rods in a magnetic field}

\section{Citation for published version (APA):}

Ven, van de, A. A. F., Tani, J., Otomo, K., \& Shindo, Y. (1987). Magnetoelastic buckling of two nearby ferromagnetic rods in a magnetic field. (RANA : reports on applied and numerical analysis; Vol. 8716). Technische Universiteit Eindhoven.

\section{Document status and date:}

Published: 01/01/1987

\section{Document Version:}

Publisher's PDF, also known as Version of Record (includes final page, issue and volume numbers)

\section{Please check the document version of this publication:}

- A submitted manuscript is the version of the article upon submission and before peer-review. There can be important differences between the submitted version and the official published version of record. People interested in the research are advised to contact the author for the final version of the publication, or visit the $\mathrm{DOI}$ to the publisher's website.

- The final author version and the galley proof are versions of the publication after peer review.

- The final published version features the final layout of the paper including the volume, issue and page numbers.

Link to publication

\section{General rights}

Copyright and moral rights for the publications made accessible in the public portal are retained by the authors and/or other copyright owners and it is a condition of accessing publications that users recognise and abide by the legal requirements associated with these rights.

- Users may download and print one copy of any publication from the public portal for the purpose of private study or research.

- You may not further distribute the material or use it for any profit-making activity or commercial gain

- You may freely distribute the URL identifying the publication in the public portal.

If the publication is distributed under the terms of Article 25fa of the Dutch Copyright Act, indicated by the "Taverne" license above, please follow below link for the End User Agreement:

www.tue.nl/taverne

Take down policy

If you believe that this document breaches copyright please contact us at:

openaccess@tue.nl

providing details and we will investigate your claim. 


\title{
EINDHOVEN UNIVERSITY OF TECHNOLOGY
}

Department of Mathematics and Computing Science

\author{
RANA $87-16$ \\ December 1987
}

MAGNETOELASTIC BUCKLING OF TWO NEARBY

FERROMAGNETIC RODS IN A MAGNETIC FIELD

by

A.A.F. van de Ven

J. Tani

K. Otomo

Y. Shindo

R(eports) A(pplied) N(umerical) A (nalysis)

Eindhoven University of Technology

Department of Mathematics and Computing Science

P.O. Box 513

5600 MB Eindhoven

The Netherlands 


\title{
MAGNETOELASTIC BUCKLING OF TWO NEARBY
}

FERROMAGNETIC RODS IN A MAGNETIC FIELD

\author{
by \\ A.A.F. van de Ven \\ Eindhoven University of Technology, \\ Department of Mathematics and Computing Science, \\ P.O. Box 513, \\ 5600 MB Eindhoven, \\ The Netherlands. \\ J. Tani, K. Otomo \\ Institute of High Speed Mechanics, \\ Tohoku University, \\ Sendai, \\ Japan. \\ Y. Shindo \\ Faculty of Engineering, \\ Tokohu University, \\ Sendai, \\ Japan.
}

With 3 Figures. 


\section{Summary}

The interactive effect of two nearby soft ferromagnetic rods on the magnetoelastic buckling is studied. The cantilevered rods are parallel to each other. The buckling is due to a uniform magnetic field normal to the axes of the rods, making an arbitrary angle with the plane through the axes of the rods. The buckling analysis is based upon a perturbation theory in which the final (or buckled) state of the system is considered as a small perturbation of an intermediate equilibrium state, for which the rigidbody state is taken. The unknown rigid-body and perturbed fields are solved with use of the theory of complex functions. The forces of magnetic origin on the deflected rods are calculated and the buckling problem is reduced to an eigenvalue problem. It is found that the interaction between the two rods considerably reduces the buckling magnetic field in case of two nearby rods. The buckling mode turns out to be symmetric. These theoretically predicted results are confirmed experimentally. Finally, the influences of the direction of the basic magnetic field and of the predeflection (before buckling) are discussed. 


\section{Introduction}

In [1], Tani and Otomo investigated the interactive effect of two soft ferromagnetic plates close to each other and placed in an external uniform magnetic field. Their results, both theoretical and experimental, reveal that the buckling magnetic field is lower for the two nearby ferromagnetic plates than for the single one. The lowest buckling mode of the two plates is a symmetric one. In the present paper the analogous problem is studied, that occurs when the two plates are replaced by two circular rods.

When a system of one or more ferromagnetic elastic bodies is placed in an external magnetic field, interaction between the magnetic and elastic fields occurs. This magnetoelastic behaviour can be described by a general set of equations and boundary conditions for the magnetic fields $\underline{H}, \underline{B}$ and $\underline{M}$ and for the displacement $\underline{u}$, as can be found, for instance, in [2]. These equations always refer to the final or deformed state of the bodies. For stability considerations it is necessary to consider this final state as a perturbation of an intermediate state, the stability of which is to be investigated. For this intermediate state the rigid-body state is taken (the fields in this state are $\underline{H}^{\circ}$ and $\underline{B}^{\circ}$ ). The general set of equations is then linearized with respect to the perturbations (indicated by small letters), which are due to the displacements in buckling of the system. This method is in close analogy with the one followed by Van de Ven in [3] or [4] for a plate and a single beam, respectively.

The thus obtained sets of equations which, after a separation of variables, are two-dimensional, will be reformulated in terms of complex functions. We shall construct a general solution in terms of series containing Besselfunctions. The coefficients in these series are determined numerically, and in this way numerical values for the buckling load are obtained for the total range of very close to faraway rods. As in [1], it turns out that the buckling value is lowered, compared to the single rod, and that the buckling is in a symmetric mode.

We have conducted a set of experiments for a system of two cantilevered rods. The experimental results are in good agreement with the theoretical ones, and they support the theoretical predictions of a lower buckling value and a symmetrical buckling mode. 


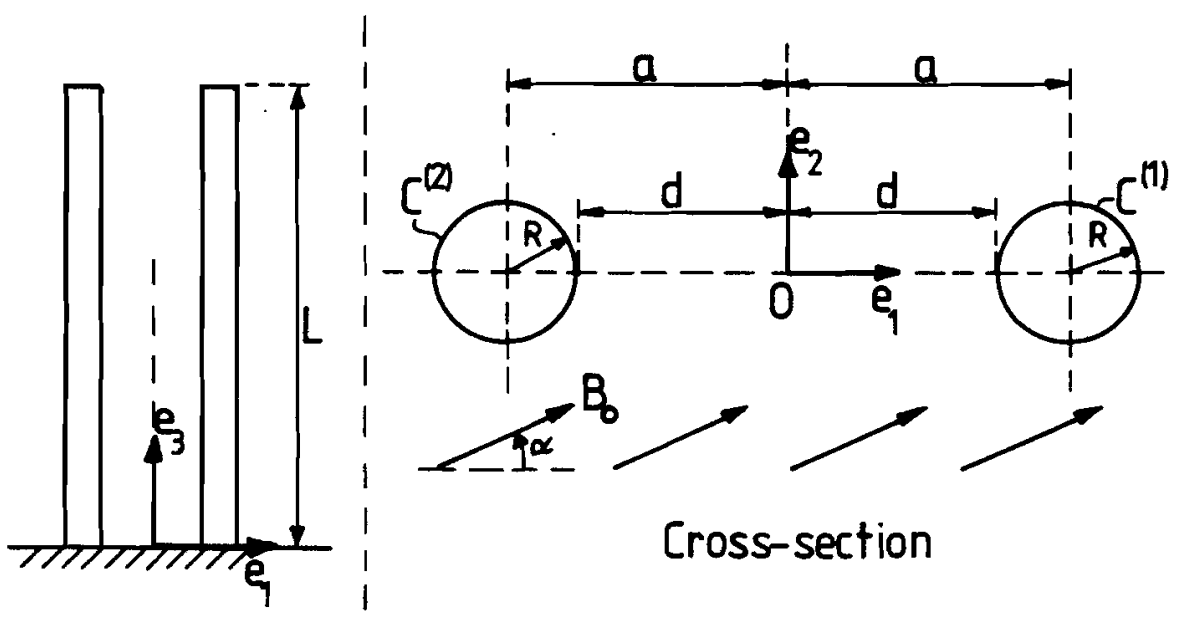

Fig. 1. System of two rods in uniform field.

Two identical soft ferromagnetic elastic rods are set parallel to each other in a static, uniform, transverse magnetic field $\underline{B}_{0}$. The circular rods have radii $R$ and lengths $l$, and their axes are a distance $2 a$ apart. The coordinate system $\left[O \underline{e}_{1} \underline{e}_{2} \underline{e}_{3}\right]$ is taken as shown in Figure 1. The cross-sections of the two rods are denoted by $G^{(1)}$ and $G^{(2)}$, with boundaries $C^{(1)}$ and $C^{(2)}$ respectively, and the vacuum by $G^{+}$. The basic field $\underline{B}_{0}$ is given by

$$
\underline{B}_{0}=B_{0} \cos \alpha \underline{e}_{1}+B_{0} \sin \alpha \underline{e}_{2}, \quad\left(\alpha \in\left(0, \frac{\pi}{2}\right)\right) .
$$

Since the rods are considered as slender, i.e., $R / l<<1$, the influences of the end sections of the rods on the magnetic field are neglected. Then the rigid-body magnetic field $\underline{H}^{0}$ can be expressed in a two-dimensional magnetic potential $\Phi\left(x_{1}, x_{2}\right)$ by

$$
\underline{H}^{0}=-\operatorname{grad} \Phi\left(x_{1}, x_{2}\right) \text {. }
$$

We denote

$$
\begin{aligned}
\Phi & =\Phi^{+}\left(x_{1}, x_{2}\right), \quad \text { for }\left(x_{1}, x_{2}\right) \in G^{+} \\
& =\Phi^{(n)}\left(x_{1}, x_{2}\right), \quad \text { for }\left(x_{1}, x_{2}\right) \in G^{(n)}, \quad n=1,2 .
\end{aligned}
$$

The rigid-body problem is then described by the following set of equations and boundary conditions $\mu_{0}$ and $\mu$ are the magnetic permeability in vacuum and in the ferromagnetic media, respectively, with 
$\left.\mu \gg \mu_{0}\right)$

$\underline{\left(x_{1}, x_{2}\right) \in G^{+}}, \quad \Delta \Phi^{+}\left(x_{1}, x_{2}\right)=0$,

$$
\Phi^{+} \rightarrow-\frac{1}{\mu_{0}}\left(\underline{B}_{0}, \underline{x}\right) \text {, as }\left(x_{1}^{2}+x_{2}^{2}\right)^{1 / 2} \rightarrow \infty ;
$$

$\underline{\left(x_{1}, x_{2}\right) \in G^{(n)}}, \quad(n=1,2)$,

$$
\Delta \Phi^{(n)}\left(x_{1}, x_{2}\right)=0
$$

$\underline{\left(x_{1}, x_{2}\right) \in C^{(n)}},(n=1,2)$,

$$
\Phi^{+}-\Phi^{(n)}=0, \quad \mu_{0} \frac{\partial \Phi^{+}}{\partial N}-\mu \frac{\partial \Phi^{(n)}}{\partial N}=0 .
$$

The perturbations on the rigid-body state are due to the deflections of the rods. Since these rods are slender, the displacement of the central line of a rod may be taken for this deflection. Thus, we obtain for the $n$th $\operatorname{rod}(n=1,2)$

$$
\underline{u}^{(n)}=\underline{u}^{(n)}\left(x_{3}\right)=u_{1}^{(n)}\left(x_{3}\right) \underline{e}_{1}+u_{2}^{(n)}\left(x_{3}\right) \underline{e}_{2} .
$$

The perturbed magnetic fields are denoted by $\underline{h}$ and $\underline{b}$ and the magnetoelastic stresses by $t_{i j}$ $(i, j \in[1,2,3])$. The equations for the perturbations are known in literature and, therefore, we refrain from deriving them here and give at once the set of linearized equations (with reference to [3] and [4]). Since the relative magnetic permeability $\mu_{r}:=\mu / \mu_{0}$ is very large, terms of $O\left(\mu_{r}^{-1}\right)$ will be neglected. Consequences of this are

- the magnetic volume force vanishes,

- the magnetoelastic stresses may be identified with the purely elastic stresses,

- the influence of the pre-stresses (i.e., $T_{i j}^{0}$ ) may be neglected.

In the usual tensor notation (including summation convention) we then have

$\underline{\underline{x}} \in G^{+}$

$$
\begin{aligned}
& b_{i, i}^{+}=0, \quad e_{i j k} h_{k, j}^{+}=0, \quad b_{i}^{+}=\mu_{0} h_{i}^{+}, \\
& b_{i}^{+} \rightarrow 0, \quad \text { as }|\underline{x}| \rightarrow \infty ;
\end{aligned}
$$


$\underline{\underline{x} \in G^{(n)}}, \quad(n=1,2), \quad($ cf [3], Eq. $(2.2))$,

$$
\begin{aligned}
e_{i j k} h_{k, j}^{(n)} & =-e_{i j k} \Phi, \Phi_{, l}^{(n)} u_{l, j}^{(n)}=-e_{i 3 k} \Phi_{, k l}^{(n)} u_{l}^{(n)}\left(x_{3}\right), \quad\left('=\frac{d}{d x_{3}}\right), \\
b_{i, i}^{(n)} & =-\mu \Phi_{, i j}^{(n)} u_{j, i}^{(n)}=0, \quad b_{i}^{(n)}=\mu h_{i}^{(n)}, \\
t_{i j, j}^{(n)} & =0,
\end{aligned}
$$

(here, use has been made of (2.7) and of $\Phi_{, 3}=0$; furthermore, the stresses $t_{i j}$ satisfy Hooke's law, but this will not be used explicitly);

$\underline{\underline{x} \in C^{(n)}}, \quad(n=1,2) \quad$ (cf. [4], Eq. (2.9))

$$
\begin{aligned}
e_{i j k}\left(h_{j}^{+}-h_{j}^{(n)}\right) N_{k} & =-e_{i j k}\left(\Phi_{, j}^{+}-\Phi_{, j}^{(n)}\right) u_{l, k}^{(n)} N_{l}-e_{i j k} \Phi_{, k l}^{+} u_{l}^{(n)} N_{j}, \\
\left(h_{i}^{+}-\mu_{r} h_{i}^{(n)}\right) N_{i} & =-\left(\Phi_{, i}^{+}-\mu_{r} \Phi_{, i}^{(n)}\right) u_{j, i}^{(n)} N_{j}+\Phi_{, j}^{+} u_{j}^{(n)} N_{i}=\Phi_{, i j}^{+} u_{j}^{(n)} N_{i}, \\
t_{i j}^{(n)} N_{j} & =-\mu_{0} \mu_{r}^{2} \frac{\partial \Phi^{(n)}}{\partial N} h_{j}^{(n)} N_{j} N_{i}=: T_{i}^{(n)},
\end{aligned}
$$

where $\underline{N}$ is the unit outward normal on $C^{(n)}$. In simplifying the right-hand side of the second boundary condition, we have used (2.7) and $\Phi_{3}=N_{3}=0$. In the same way the first boundary condition can be reduced to yield

$$
\begin{aligned}
h_{3}^{+}-h_{3}^{(n)} & =\mu_{r} \frac{\partial \Phi^{(n)}}{\partial N} u_{i}^{(n) \rho} N_{i}, \\
e_{3 j k}\left(h_{j}^{+}-h_{j}^{(n)}\right) N_{k} & =-e_{3 j k} \Phi_{, i k}^{+} u_{i}^{(n)} N_{j} .
\end{aligned}
$$

Although the perturbed problem is essentially a three-dimensional problem, it can be trasformed into a two-dimensional problem by a separation of variables (comparable with the substitutions (4.1) in [4]). Such two-dimensional problems can most elegantly be solved by making use of the theory of complex functions. In the next section a formulation in terms of complex functions of the present problem will be presented. 


\section{Formulation in terms of complex functions}

\subsection{The rigid-body problem}

Since $\Phi\left(x_{1}, x_{2}\right)$ is a harmonic function, there exists an analytical function $F(z)$, with $z=x_{1}+i x_{2}$, such that

$$
\Phi=\operatorname{Re} F(z), \quad z \in \boldsymbol{C} \backslash C^{(1)} \cup C^{(2)}
$$

The imaginary part of $F(z)$ is the conjugate function $\Psi$ of $\Phi$, i.e.

$$
\operatorname{Im} F(z)=\Psi, \quad \text { and } \quad \frac{\partial \Phi}{\partial x_{1}}=\frac{\partial \Psi}{\partial x_{2}}, \quad \frac{\partial \Phi}{\partial x_{2}}=-\frac{\partial \Psi}{\partial x_{1}}
$$

which is also a harmonic function.

In terms of $\Psi$ the second boundary condition of (2.6) reads (up to an arbitrary constant)

$\underline{z \in C^{(n)}}, \quad \Psi^{+}-\mu_{r} \Psi^{(n)}=0, \quad(n=1,2)$.

The boundary conditions (2.6) ${ }^{1}$ and (3.3) can now easily be expressed in $F(z)$. This yields

$\underline{z \in C^{(n)}}, \quad F^{+}+\bar{F}^{+}-F^{(n)}-\bar{F}^{(n)}=0$,

$$
F^{+}-\bar{F}^{+}-\mu_{r} F^{(n)}+\mu_{r} \bar{F}^{(n)}=0
$$

where $\bar{F}=\Phi-i \Psi$, the complex conjugate of $F$. Together with the condition at infinity (from $(2.4)^{2}$ )

$$
F^{+}(z) \rightarrow-\frac{1}{\mu_{0}} B_{0} z e^{-i \alpha}, \text { as }|z| \rightarrow \infty \text {, }
$$

the boundary conditions (3.4) completely determine the analytical function $F(z)$. The explicit solution will be given in the next section.

\subsection{The perturbations}

We introduce the complex displacements $w^{(n)}\left(x_{3}\right), n=1,2$, by

$$
w^{(n)}\left(x_{3}\right)=u_{1}^{(n)}\left(x_{3}\right)+i u_{2}^{(n)}\left(x_{3}\right)
$$

It can be shown that the solutions of (2.8) and (2.9) can be expressed in $w^{(n)}\left(x_{3}\right)$ and two complex functions $\zeta_{1}(z, \bar{z})$ and $\zeta_{2}(z, \bar{z})$ by 
$\underline{z \in G^{+}}$

$$
\begin{aligned}
h_{1}^{+}-i h_{2}^{+} & =2 \sum_{m=1}^{2}\left[\frac{\partial \zeta_{m}^{+}}{\partial z} w^{(m)}+\frac{\partial \bar{\zeta}_{m}^{+}}{\partial z} \bar{w}^{(m)}\right], \\
h_{3}^{+} & =2 \sum_{m=1}^{2}\left[\zeta_{m}^{+} \frac{d w^{(m)}}{d x_{3}}+\bar{\zeta}_{m}^{+} \frac{d \bar{w}^{(m)}}{d x_{3}}\right]
\end{aligned}
$$

$\underline{z \in G^{(n)}}$

$$
\begin{aligned}
h_{1}^{(n)}-i h_{2}^{(n)} & =-\frac{d^{2} F^{(n)}}{d z^{2}} w^{(n)}+2 \sum_{m=1}^{2}\left[\frac{\partial \zeta_{m}^{(n)}}{\partial z} w^{(m)}+\frac{\partial \bar{\zeta}_{m}^{(n)}}{\partial z} \bar{w}^{(m)}\right], \\
h_{3}^{(n)} & =2 \sum_{m=1}^{2}\left[\zeta_{m}^{(n)} \frac{d w^{(m)}}{d x_{3}}+\bar{\zeta}_{m}^{(n)} \frac{d \bar{w}^{(m)}}{d x_{3}}\right] .
\end{aligned}
$$

This substitution constitutes a solution for (2.8) - (2.9) only if $\zeta_{m}(z, \bar{z})$ and $w^{(n)}\left(x_{3}\right)$ satisfy (for $z \in \mathbb{C} \backslash C^{(1)} \cup C^{(2)}$ )

$$
4 \frac{\partial^{2} \zeta_{m}}{\partial z \partial \bar{z}}-\lambda^{2} \zeta_{m}=0, \quad(m=1,2)
$$

and

$$
\frac{d^{2} w^{(n)}}{d x_{3}^{2}}+\lambda^{2} w^{(n)}=0, \quad(n=1,2)
$$

The parameter $\lambda$ is determined by the support conditions of the rods. Here, it is assumed that the support conditions are isotropic in the $\underline{e}_{1}-\underline{e}_{2}$-plane and identical for both rods: then $\lambda \in \mathbb{R}$ and $\lambda^{(1)}=\lambda^{(2)}$. In case of a cantilever we have $\lambda=\pi / 2 l$.

We note that (3.9) is the complex representation of the wave or Helmholtz-equation and that (3.10) is the necessary condition assuring that the separation according to (3.7) - (3.8) is consistent with (2.8) and (2.9) (compare this with Equations (4.3) and (4.4) in [4], for a single rod).

With

$$
N_{1} \pm i N_{2}=e^{ \pm i \phi} \text {, and } \frac{\partial}{\partial N}=\frac{\partial}{\partial r}, \text { at } C^{(n)},
$$

and with the substitutions (3.7) and (3.8) the following boundary conditions for $\zeta_{1}(z, \bar{z})$ can be derived from $(2.10)^{2}$ and (2.11). 
$\underline{z \in C^{(1)}}$

$$
\begin{array}{r}
\zeta_{1}^{+}-\zeta_{1}^{(1)}=\frac{\mu_{r}}{4}\left[\frac{d F^{(1)}}{d z}+e^{-2 i \phi} \frac{d \bar{F}^{(1)}}{d \bar{z}}\right], \\
\frac{\partial \zeta_{1}^{+}}{\partial r}-\mu_{r} \frac{\partial \zeta_{1}^{(1)}}{\partial r}=\frac{1}{2} e^{i \phi}\left[\frac{d^{2} F^{+}}{d z^{2}}-\mu_{r} \frac{d^{2} F^{(1)}}{d z^{2}}\right]
\end{array}
$$

\section{$\underline{z \in C^{(2)}}$}

$$
\zeta_{1}^{+}-\zeta_{1}^{(2)}=0,
$$

$$
\frac{\partial \zeta_{1}^{+}}{\partial r}-\mu_{r} \frac{\partial \zeta_{1}^{(2)}}{\partial r}=0 \text {. }
$$

These boundary conditions must be supplemented by the condition at infinity

$$
\zeta_{1}^{+}(z, \bar{z}) \rightarrow 0, \quad \text { as }|z| \rightarrow \infty \text {. }
$$

The explicit solution for $\zeta_{1}(z, \bar{z})$ can be written as a series in Besselfunctions as we shall show in Section 6 . Once $\zeta_{1}$ is determined $\zeta_{2}$ follows from the symmetry relation

$$
\zeta_{2}(z, \bar{z})=\zeta_{1}(-z,-\bar{z})
$$




\section{The beam equations}

The bending of a slender rod is governed by the one-dimensional beam equation

$$
E I \frac{d^{4} w^{(n)}}{d x_{3}^{4}}=q^{(n)}\left(x_{3}\right), \quad(n=1,2),
$$

where $E I$ is the bending stiffness of the circular rod ( $E$ is Young's modulus and $\left.I=\pi R^{4} / 4\right)$. In the absence of volume forces (see $\left.(2.9)^{4}\right)$ the normal load per unit of length $q^{(n)}\left(x_{3}\right)$ is given by

$$
\left.q^{(n)}\left(x_{3}\right)=\int_{C^{(n)}}\left(T_{1}^{(n)}+i T_{2}^{(n)}\right) d s=-\mu_{0} \mu_{r}^{2} \int_{C^{(n)}} \frac{\partial \Phi^{(n)}}{\partial N} \underline{h}^{(n)} \underline{N}\right) e^{i \phi} d s,
$$

where $T_{1}^{(n)}$ and $T_{2}^{(n)}$ are taken according to $(2.10)^{3}$.

The right-hand side of (4.2) can be written as a linear combination of $w^{(1)}, \bar{w}^{(1)}, w^{(2)}$ and $\bar{w}^{(2)}$. Hence, the beam equation (4.1) has in general only the trivial solution $w^{(1)}=w^{(2)}=0$. Only for special values of $B_{0}$ (note that $q^{(n)}$ depends on $B_{0}$ ) (4.1) has a non-trivial solution. We are interested in the lowest of these values, which we shall call the critical or buckling value of $B_{0}$. 


\section{Solution for the rigid-body problem}

Since $F(z)$ is analytical for $z \in \mathbb{C} \backslash C^{(1)} \cup C^{(2)}$, it can be represented as Laurent-series in $(z \pm a)$. This implies the following formulation which satisfies the condition (3.5), the boundary condition (3.4) (for $\mu_{r} \gg 1$ ) and the obvious symmetry relation $F(-z)=-F(z)$,

$$
\begin{array}{rlr}
F(z) & =F^{(1)}(z)=a^{(0)}-\frac{2}{\mu_{r}} \sum_{n=1}^{\infty} \bar{c}_{n}\left[\frac{z-a}{R}\right]^{n}, & \text { for } z \in G^{(1)}, \\
& =F^{(2)}(z)=-F^{(1)}(-z), & \text { for } z \in G^{(2)}, \\
& =F^{+}(z)=-\frac{1}{\mu_{0}} B_{0} z e^{-i \alpha}+\sum_{n=1}^{\infty} c_{n}\left\{\left[\frac{R}{z-a}\right]^{n}-(-1)^{n}\left[\frac{R}{z+a}\right]^{n} \text { for } z \in G^{+} .\right.
\end{array}
$$

The still unknown coefficients $c_{n}\left(a^{(0)}\right.$ is an irrelevant constant) can be determined from the only remaining boundary condition $(3.4)^{1}$. We write

$$
c_{n}=\frac{1}{\mu_{0}} B B_{0} e^{i \alpha}\left(\delta_{n 1}+\gamma_{n} \delta^{n+1}\right), \quad(n=1,2, \ldots),
$$

where $\delta_{i j}$ is Kronecker's delta and

$$
\delta=\frac{R}{2 a}, \quad\left(0<\delta<\frac{1}{2}\right)
$$

Then, (3.4) ${ }^{1}$ implies the recurrence relations for $\gamma_{n}$ (with $\mu_{r}^{-1} \approx 0$ )

$$
\gamma_{n}-(-1)^{n} e^{-2 i \alpha} \sum_{m=1}^{\infty}(-1)^{m}\left[\begin{array}{c}
n+m-1 \\
n
\end{array}\right] \delta^{2 m} \bar{\gamma}_{m}=(-1)^{n+1} e^{-2 i \alpha}
$$

The solution of this set reads

$$
\gamma_{n}=-\frac{1}{\delta} \sum_{m=1}^{\infty}\left(\cos \alpha+(-1)^{m} i \sin \alpha\right) p_{m} q_{m}^{n} e^{-i \alpha}, \quad(n \geq 1),
$$

where

$$
p_{m}=\frac{\left(1-d^{2}\right)^{2} d^{2 m-1}}{\left(1-d^{2 m}\right)\left(1-d^{2 m+2}\right)}, \quad q_{m}=-\frac{\left(1-d^{2 m}\right)}{\left(1-d^{2 m+2}\right)}\left(\frac{d}{\delta}\right)
$$

and

$$
d=\frac{2 \delta}{1+\sqrt{1-4 \delta^{2}}}, \quad(0<d<1)
$$




\section{Solution for the perturbations}

With the solution (5.1) for $F(z)$ the boundary conditions (3.12) become

$\underline{z \in C^{(1)}}$

$$
\begin{array}{r}
\zeta_{1}^{+}-\zeta_{1}^{(1)}=\sum_{n=-\infty}^{\infty} R_{n} e^{-i n \phi}, \\
\frac{\partial \zeta_{1}^{+}}{\partial r}-\mu_{r} \frac{\partial \zeta_{1}^{(1)}}{\partial r}=\sum_{n=-\infty}^{\infty} S_{n} e^{-i n \phi},
\end{array}
$$

where (for $\mu_{r} \gg 1$ )

$$
\begin{aligned}
R_{n} & =-\frac{(n-1)}{2 R} c_{n-1}, & & n \geq 2, \\
& =0, & & n=1, \\
& =-\frac{(-n+1)}{2 R} \bar{c}_{1-n}, & & n \leq 0,
\end{aligned}
$$

and

$$
S_{n}=-\ln \mid R_{n} .
$$

In order to satisfy these boundary conditions, we represent $\zeta_{1}$ in terms of $e^{i n \phi}$. Then, the general solution of the wave equation (3.9), that satisfies the radiation condition (3.14) and remains finite inside $G^{(1)}$ and $G^{(2)}$, can be written as

$\underline{z \in G^{(1)}}$

$$
\zeta_{1}=\zeta_{1}^{(1)}=\sum_{n=-\infty}^{\infty} D_{n} \frac{I_{n}(\lambda r)}{I_{n}(\lambda R)} e^{-i n \phi}, \quad\left(z=a+r e^{i \phi} ; 0<r<R\right)
$$

$\underline{z \in G^{(2)}}$

$$
\zeta_{1}=\zeta_{1}^{(2)}=\sum_{n=-\infty}^{\infty} E_{n} \frac{I_{n}(\lambda r)}{I_{n}(\lambda R)} e^{-i n \phi}, \quad\left(z=-a-r e^{i \phi} ; 0 \leq r<R\right)
$$

$\underline{z \in G^{+}}$

$$
\zeta_{1}=\zeta_{1}^{+}=\sum_{n=-\infty}^{\infty}\left\{F_{n} \frac{K_{n}\left(\lambda S_{1}\right)}{K_{n}(\lambda R)} e^{-i n \Phi_{1}}+G_{n} \frac{K_{n}\left(\lambda S_{2}\right)}{K_{n}(\lambda R)} e^{-i n \Phi_{2}}\right\}
$$

where

$$
S_{1} e^{i \Phi_{1}}=z-a, \text { and } S_{2} e^{i \Phi_{2}}=-z-a,\left(\text { for } z \in G^{+}\right)
$$


Assume that $z$ is in the neighbourhood of $C^{(1)}$ (say $\left.0 \leq|z-a| \leq a\right)$ and put $z=a+r e^{i \phi}$. Then

$$
S_{1} e^{i \Phi_{1}}=r e^{i \phi} \text {, and } S_{2} e^{i \Phi_{2}}=-2 a-r e^{i \phi}
$$

For the further evaluation of the second term on the right-hand side of (6.6) we use a theorem from the theory of Besselfunctions which states (cf. [5], Eq. (2.33), page 36)

for $R<r<a$,

$$
K_{n}\left(\lambda S_{2}\right) e^{-i k \Phi_{2}}=\sum_{m=-\infty}^{\infty}(-1)^{m+n} K_{m+n}(2 a \lambda) I_{m}(\lambda r) e^{i m \phi} .
$$

Substituting (6.8) and (6.9) into (6.6) and interchanging the order of summation, we arrive at (note that $I_{-n}=I_{n}$ and $K_{-n}=K_{n}$ )

$$
\zeta_{1}^{+}=\sum_{n=-\infty}^{\infty}\left\{F_{n} \frac{K_{n}(\lambda r)}{K_{n}}+\frac{I_{n}(\lambda r)}{I_{n}} \sum_{m=-\infty}^{\infty} K_{m n} G_{-m}\right\} e^{-i n \phi},
$$

holding for $z=a+r e^{i \phi} \in G^{+}, R<r<a$. In (6.10) we have written $I_{n}$ and $K_{n}$ for $I_{n}(\lambda R)$ and $K_{n}(\lambda R)$, respectively, and we have introduced

$$
K_{m n}=\frac{I_{n}}{K_{m}} K_{m+n}(2 a \lambda) .
$$

It is now a straightforward procedure to determine $D_{n}, E_{n}, F_{n}$ and $G_{n}$ from the boundary conditions (6.1) and (3.13). With the definitions

$$
\tau_{n}=\mu \lambda R I_{n}^{\prime} K_{n}+1 ; T_{n}=\lambda R I_{n}^{\prime} K_{n} R_{n}-I_{n} K_{n} S_{n}
$$

we thus obtain

$$
\begin{aligned}
& F_{n}=-\left(\tau_{n}-1\right) \hat{D}_{n}, \quad G_{n}=-\left(\tau_{n}-1\right) E_{n}, \\
& E_{n}=-\sum_{m=-\infty}^{\infty}(-1)^{m+n} \frac{\left(\tau_{m}-1\right)}{\tau_{n}} K_{m n} \hat{D}_{-m}, \quad D_{n}=\hat{D}_{n}+\frac{T_{n}}{\left(\tau_{n}-1\right)},
\end{aligned}
$$

where $\hat{D}_{n}$ is the solution of

$$
\hat{D}_{n}-(-1)^{n} \sum_{m=-\infty}^{\infty} \Gamma_{n n} \hat{D}_{m}=\Lambda_{n}, \quad(n \in \mathbb{Z})
$$

with

$$
\Gamma_{n m}=(-1)^{m}\left[\frac{\tau_{m}-1}{\tau_{n}}\right] \sum_{r=-\infty}^{\infty}\left[\frac{\tau_{r}-1}{\tau_{r}}\right] K_{m r} K_{r n},
$$


and

$$
\Lambda_{n}=\frac{T_{n}-R_{n}}{\tau_{n}}-\frac{T_{n}}{\tau_{n}-1}
$$

The infinite system (6.14) can be solved numerically after truncation. The coefficients $D_{n}, E_{n}, F_{n}$ and $G_{n}$ can then be calculated from (6.13). The solution for $\zeta_{1}(z, \bar{z})$ is thus completely determined. The complementary function $\zeta_{2}(z, \bar{z})$ follows from the symmetry condition (3.15).

\section{NOTE}

The above results simplify considerably when the slenderness of the rods is taken into account (i.e., $\lambda R<\lambda a<<1$ ). We have used these simplifications in our numerical evaluation, but we shall not enter into the details here. 


\section{The buckling equation}

The results of the preceding two sections enable us to work out the relation (4.2) for the magnetic load $q^{(n)}\left(x_{3}\right)$ explicitly in terms of $w^{(1)}$ and $w^{(2)}$.

With (3.1) and with $F^{(1)}(z)$ according to (5.1), we have for $z \in C^{(1)}$,

$$
\begin{aligned}
& e^{i \phi} \frac{d \Phi^{(1)}}{d N}=\frac{1}{2}\left[e^{2 i \phi} \frac{d F^{(1)}}{d z}+\frac{d \bar{F}^{(1)}}{d \bar{z}}\right]= \\
& =-\frac{1}{\mu_{r} R}\left\{\sum_{m=1}^{\infty}(m-1) \bar{c}_{m-1} e^{i m \phi}+\sum_{m=0}^{\infty}(m+1) c_{m+1} e^{-i m \phi}\right\} .
\end{aligned}
$$

Furthermore, starting from (3.8) we deduce, for $z \in C^{(1)}$,

$$
\left(\underline{h}^{(1)}, \underline{N}\right)=\frac{\partial}{\partial r} \sum_{m=1}^{2}\left[\zeta_{m}^{(1)} w^{(m)}+\bar{\zeta}_{m}^{(1)} \bar{w}^{(m)}\right]-\frac{1}{2}\left[e^{i \phi} \frac{d^{2} F^{(1)}}{d z^{2}} w^{(1)}+e^{-i \phi} \frac{d^{2} \bar{F}^{(1)}}{d \bar{z}^{2}} \bar{w}^{(1)}\right] .
$$

The symmetry relation (3.15) implies

$$
\zeta_{2}^{(1)}(z, \bar{z})=\zeta_{1}^{(2)}(-z,-\bar{z}), \quad\left(\text { for } z \in C^{(1)}\right) .
$$

The use of (7.3), (6.4), (6.5) and (5.1) in (7.2) results in

$$
\left(h^{(1)}, \underline{N}\right)=\frac{1}{R}\left\{\sum_{n=0}^{\infty} H_{n} e^{-i n \phi}+\sum_{n=1}^{\infty} \bar{H}_{n} e^{i n \phi}\right\}
$$

where

$$
H_{n}=\frac{\lambda R I_{n}^{\prime}}{I_{n}}\left\{D_{n} w^{(1)}+\bar{D}_{-n} \bar{w}^{(1)}+E_{n} w^{(2)}+\bar{E}_{-n} \bar{w}^{(2)}\right\}+\frac{n(n+1)}{\mu_{r} R} c_{n+1} \bar{w}^{(1)} .
$$

Substituting (7.1) and (7.4) into (4.2) (for $n=1$ ), using (5.2) for $c_{n}$ and performing the integration over $C^{(1)}$, we finally arrive at

$$
q^{(1)}\left(x_{3}\right)=2 \pi \mu B_{0}\left\{H_{0} e^{i \alpha}+H_{2} e^{-i \alpha}+\sum_{n=1}^{\infty} n \delta^{n+1}\left[\bar{\gamma}_{n} H_{n+1} e^{-i \alpha}+\gamma_{n} \bar{H}_{n-1} e^{i \alpha}\right]\right\} .
$$

At this point we recapitulate that once the systems (5.4) and (6.14) are (numerically) solved, the coefficients $c_{n}, D_{n}$ and $E_{n}$ are known quantities. Then, (7.5) delivers us an explicit expression for $H_{n}$ as a linear function in $w^{(1)}$ and $w^{(2)}$. Using this result in (7.6), we can rewrite this relation in a form more suitable for our further manipulations, i.e.,

$$
q^{(1)}\left(x_{3}\right)=B_{0}^{2}\left[Q_{1} w^{(1)}+Q_{2} \bar{w}^{(1)}+Q_{3} w^{(2)}+Q_{4} \bar{w}^{(2)}\right] .
$$

We note that the coefficients $Q_{n}, n=1, \ldots, 4$, are complex numbers, depending on given values for $\alpha, \delta$ and $\lambda$, but independent of $B_{0}$. 
For the load $q^{(2)}$ on the second rod a relation analogous to (7.7) holds. We merely have to interchange $w^{(1)}$ and $w^{(2)}$, so

$$
q^{(2)}\left(x_{3}\right)=B_{0}^{2}\left[Q_{3} w^{(1)}+Q_{4} \bar{w}^{(1)}+Q_{1} w^{(2)}+Q_{2} \bar{w}^{(2)}\right] .
$$

Substituting (7.7) and (7.8) into the beam equations (4.1) for $n=1$ and $n=2$, respectively, and using (as follows from (3.10))

$$
\frac{d^{4} w^{(n)}}{d x_{3}^{4}}=\lambda^{4} w^{(n)}, \quad(n=1,2),
$$

we obtain two homogeneous equations for the complex displacements $w^{(1)}$ and $w^{(2)}$ of the form

$$
\begin{aligned}
& \left(Q_{1}-\Omega\right) w^{(1)}+Q_{2} \bar{w}^{(1)}+Q_{3} w^{(2)}+Q_{4} \bar{w}^{(2)}=0, \\
& Q_{3} w^{(1)}+Q_{4} \bar{w}^{(1)}+\left(Q_{1}-\Omega\right) w^{(2)}+Q_{2} \bar{w}^{(2)}=0,
\end{aligned}
$$

where

$$
\Omega=\frac{E I \lambda^{4}}{B_{0}^{2}}
$$

Using (7.10) we can construct two independent sets of equations; one for $\left(w^{(1)}-w^{(2)}\right)$ and $\left(\bar{w}^{(1)}-\bar{w}^{(2)}\right)$ and one for $\left(w^{(1)}+w^{(2)}\right)$ and $\left(\bar{w}^{(1)}+\bar{w}^{(2)}\right)$. To this end we subsequently subtract and add the two equations (7.10) and take the complex conjugate of the result. Thus, we obtain as the first set

$$
\begin{aligned}
& \left(Q_{1}-Q_{3}-\Omega\right)\left(w^{(1)}-w^{(2)}\right)+\left(Q_{2}-Q_{4}\right)\left(\bar{w}^{(1)}-\bar{w}^{(2)}\right)=0, \\
& \left(\bar{Q}_{2}-\bar{Q}_{4}\right)\left(w^{(1)}-w^{(2)}\right)+\left(\bar{Q}_{1}-\bar{Q}_{3}-\Omega\right)\left(\bar{w}^{(1)}-\bar{w}^{(2)}\right)=0,
\end{aligned}
$$

and as the second one

$$
\begin{aligned}
& \left(Q_{1}+Q_{3}-\Omega\right)\left(w^{(1)}+w^{(2)}\right)+\left(Q_{2}+Q_{4}\right)\left(\bar{w}^{(1)}+\bar{w}^{(2)}\right)=0, \\
& \left(\bar{Q}_{2}+\bar{Q}_{4}\right)\left(w^{(1)}+w^{(2)}\right)+\left(\bar{Q}_{1}+\bar{Q}_{3}-\Omega\right)\left(\bar{w}^{(1)}+\bar{w}^{(2)}\right)=0 .
\end{aligned}
$$

What we are looking for are those eigenvalues of $\Omega$ for which one of these sets has a non-trivial solution. Assuming that the eigenvalues of the two sets do not coincide, we conclude that there remain two possibilities:

1) if $\Omega$ is an eigenvalue of (7.13), then (7.12) implies

$$
w^{(1)}=-w^{(2)}
$$

to which we refer as a "symmetrical buckling mode";

2) if $\Omega$ is an eigenvalue of (7.12), then (7.13) implies

$$
w^{(1)}=w^{(2)}
$$

which we shall call an "anti-symmetrical buckling mode". 
Our numerical results reveal that the lowest buckling value for $B_{0}$ is associated with the symmetrical buckling mode. Therefore, we put $w^{(2)}=-w^{(1)}$ in (8.10) to obtain

$$
\begin{aligned}
& \left(Q_{1}-Q_{3}-\Omega\right) w^{(1)}+\left(Q_{2}-Q_{4}\right) \bar{w}^{(1)}=0, \\
& \left(\bar{Q}_{2}-\bar{Q}_{4}\right) w^{(1)}+\left(\bar{Q}_{1}-\bar{Q}_{3}-\Omega\right) \bar{w}^{(1)}=0 .
\end{aligned}
$$

This system has only then a non-trivial solution when $\Omega$ satisfies the equation

$$
\left(Q_{1}-Q_{3}-\Omega\right)\left(\bar{Q}_{1}-\bar{Q}_{3}-\Omega\right)-\left(Q_{2}-Q_{4}\right)\left(\bar{Q}_{2}-\bar{Q}_{4}\right)=0 .
$$

Solving this equation we obtain for the highest $\Omega$-value (corresponding to the lowest $B_{0}$-value)

$$
\mathbf{\Omega}_{c r}=\operatorname{Re}\left(Q_{1}-Q_{3}\right)+\sqrt{\left|Q_{2}-Q_{4}\right|^{2}-\left[\operatorname{Im}\left(Q_{1}-Q_{3}\right)\right]^{2}} .
$$

Finally, the critical buckling value for $B_{0}$ is then found from

$$
\left(B_{0}\right)_{c r}=\lambda^{2} \sqrt{\frac{E T}{\Omega_{c r}}} .
$$




\section{Description of the experiment}

Experiments were conducted for circular rods, diameter $3 \mathrm{~mm}$ and length $150 \mathrm{~mm}$, made of mild steel, with Young's modulus $E=2.1 \times 10^{11} \mathrm{~Pa}$ and relative permeability $\mu_{r}=300$. Two such rods were clamped at one end in a brass vice parallel to each other. The uniform magnetic field was generated by an electromagnet and its intensity was controlled by a direct current supply. The tops of the rods were round up in order to minimize edge effects. The pole was circular and its diameter was 250 $\mathrm{mm}$. The gap distance between the two pole faces was $100 \mathrm{~mm}$. The magnetic field between the pole faces was measured with a gaussmeter; the deflection at a central point of a rod with an apparatus exerting negligible small contact-forces. The magnetic field and the deflection were recorded with an $X-Y$-recorder (see Fig. 3).

In these experiments the direction of the plane through the two rods (i.e. the $\underline{e}_{1}-\underline{e}_{3}$-plane) could be varied with respect to the direction of the uniform basic field $\underline{B}_{0}$, yielding variable $\alpha$-values (see Fig. 1). Experiments were conducted for two values of $\alpha$, knowing $\alpha=0$ and $\alpha=\pi / 2$ corresponding with a $\underline{B}_{0}$-field parallel to the plane of the rods and one normal to it, respectively. Furthermore, tests were done for various values of the distance between the rods. The experimental procedure for the determination of the buckling field was the same as described in [1]. 


\section{Comparison of experimental and numerical results}

The theoretical results of Section 7 were numerically evaluated, with use of the following values for the parameters

$$
R=1.5 \mathrm{~mm}, \quad l=150 \mathrm{~mm}, E=2.1 \times 10^{11} \mathrm{~Pa},
$$

and, moreover, for $\alpha=0$ or $\pi / 2$, for two values of $\mu_{r}$, i.e. $\mu_{r}=300$ or $\mu_{r}=5 \times 10^{4}$, and for $\delta$ varying from 0.1 to 0.35 . The results are listed in Table 1 .

\begin{tabular}{|c||c|c|c|c|}
\hline \multicolumn{1}{|c||}{$\delta$} & \multicolumn{4}{c|}{$B_{c}$ in Tesla $\times 10^{-2}$} \\
\cline { 2 - 5 } & $\alpha=0, \mu_{r}=300$ & $\alpha=\pi / 2, \mu_{r}=300$ & $\alpha=0, \mu_{r}=5 \times 10^{4}$ & $\alpha=\pi / 2, \mu_{r}=5 \times 10^{4}$ \\
\hline \hline 0.10 & 22.3 & 23.3 & 6.84 & 7.14 \\
0.15 & 20.3 & 22.2 & 6.05 & 6.65 \\
0.20 & 16.9 & 19.6 & 5.31 & 6.31 \\
0.25 & 13.0 & 16.1 & 4.56 & 6.03 \\
0.30 & 9.36 & 12.7 & 3.79 & 5.79 \\
0.35 & 6.41 & 9.98 & 3.00 & 5.57 \\
\hline
\end{tabular}

TABLE 1. Buckling values.

Experiments were conducted for $\mu_{r}=300$ and for $\alpha=0$ and $\alpha=\pi / 2$. For each case two distinct tests were made. The experimental results are shown in Fig. 2, together with the theoretical lines. Here, $d=a-R$ and $B_{c 2}$ and $B_{c 1}$ are the buckling fields for two rods and for one rod, respectively.
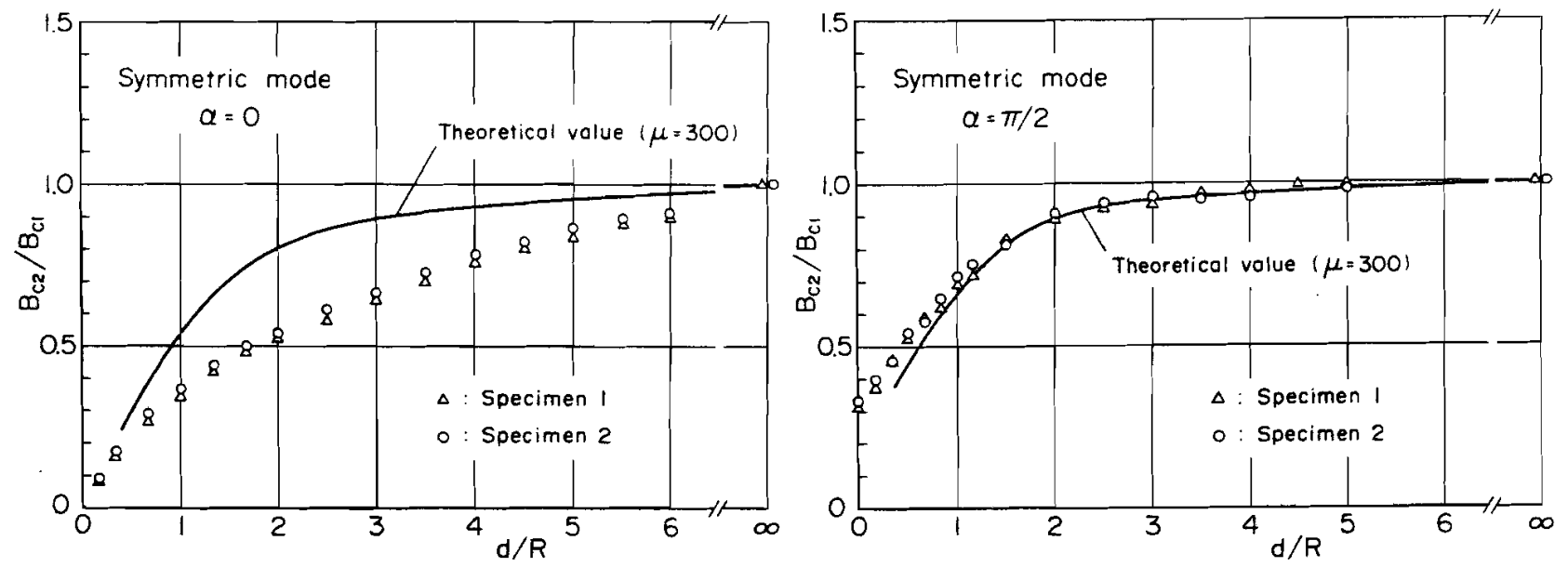

Fig. 2. Buckling magnetic field vs. distance between two rods. 
The value of $B_{c 1}$ is both experimentally determined as well as calculated from the formula (cf. [4], eq. (7.1))

$$
B_{c 1}=\frac{1}{2} \lambda R \sqrt{\frac{\mu_{0} E}{\mu_{r}}} \sqrt{1+\frac{1}{2} \mu_{r}(\lambda R)^{2} K_{0}(\lambda R)}
$$

where

$$
\lambda R=\frac{\pi R}{2 l}, \quad \text { and } \mu_{0}=4 \pi \times 10^{-7} \mathrm{H} / \mathrm{m} .
$$

With use of the values given in (8.1), the formula (8.2) yields $B_{c 1}=0,25 T$, while the experiments indicate that $B_{c 1}=0,24 T$, and, hence, these results are in good agreement with each other. The experimental value of $B_{c 1}$ can be read off from Fig. 3, showing the deflection of a rod under increasing field magnitude $B_{0}$.
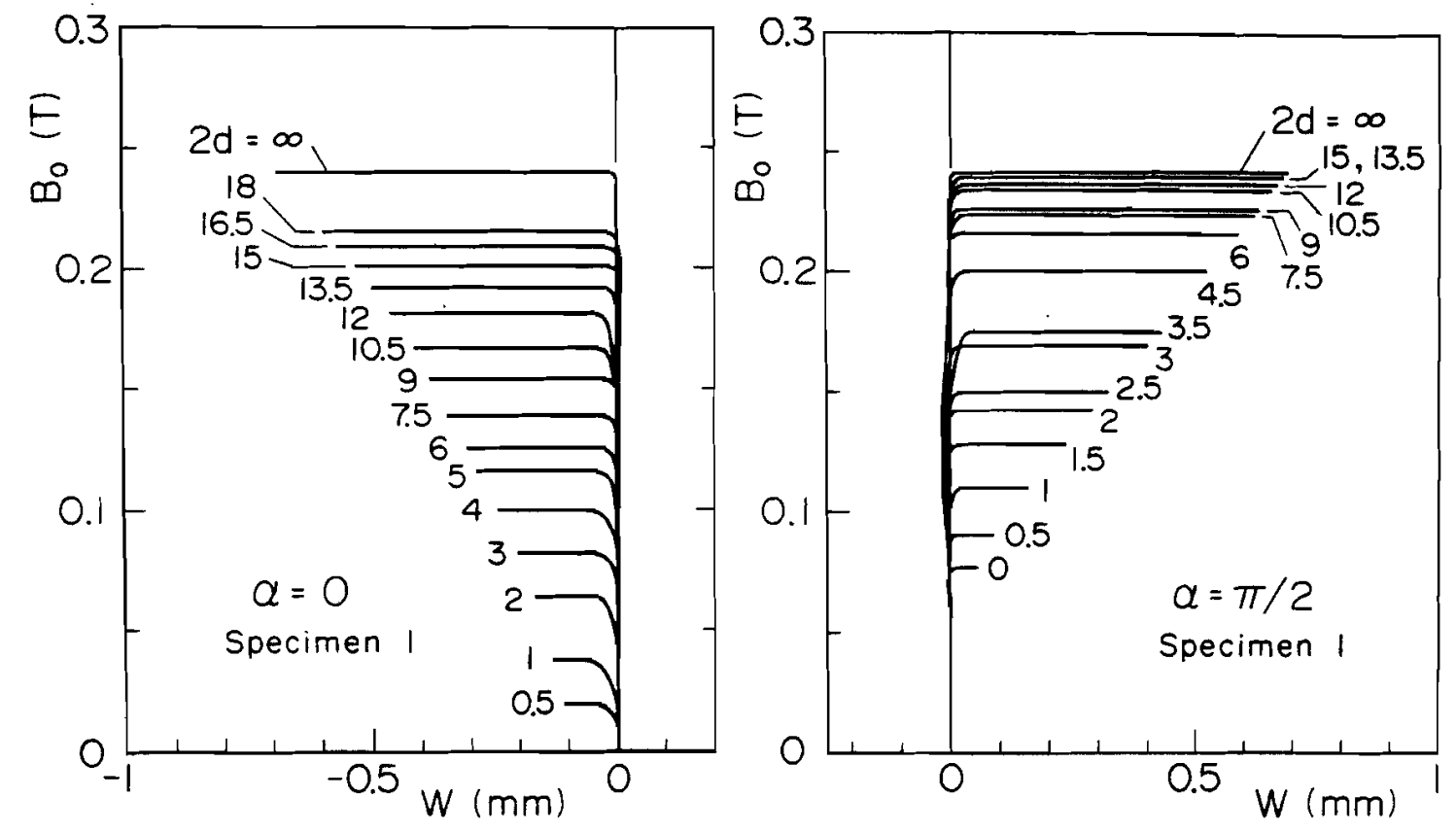

Fig. 3. Magnetic field vs. deflection.

The graphs in Fig. 2 show a reasonable correspondence between theoretical and experimental results, although this correspondence is much better in the case $\alpha=\pi / 2$ than in the case $\alpha=0$ (a possible explanation for this will be given in Section 10). Definitely, the graphs confirm our theoretical prediction of a decrease in the buckling values with decreasing distance between the rods. Furthermore, it was observed in the experiments that the buckling of the rods occurred in a symmetrical mode as was predicted in Section 7. In case $\alpha=0$ the two rods approached each other in buckling. In theory, however, there is no preference for buckling towards or away from each other, but in Section 10 we shall show that this preference is due to the prebuckling deflection of the rods (which is not taken into account in our theoretical derivations). Finally, we note that the results presented in Table 1 show that 


$$
-19-
$$

i) the buckling values for $\alpha=0$ are lower than those for $\alpha=\pi / 2$;

ii) the buckling values decrease with increasing value of $\mu_{r}$. However, it should be noted that for very large values of $\mu_{r}$ (e.g. $\mu_{r}>10^{4}$ ) the buckling value becomes practically independent of $\mu_{r}$. 


\section{Discussion}

In our analysis we have consequently neglected the influence of the prebuckling deflections. Although their influence on the buckling values is always a second-order effect, it nevertheless is worthwhile to take them into account for an explanation of some peculiarities already noticed in Section 9.

In the intermediate or pre-buckled state there acts on the boundary $C^{(1)}$ of the first rod a (normal) stress vector $\underline{T}^{(1)}$ given by (cf. [2], eq. (2.85) with $\chi \approx \mu_{r} \gg 1$ )

$$
\underline{T}^{(1)}=\frac{1}{2} \mu_{0}(\underline{M}, \underline{N})^{2} \underline{N}=\frac{1}{2} \mu_{0} \mu_{r}^{2}\left[\frac{\partial \Phi^{(1)}}{\partial N}\right]^{2} \underline{N},
$$

where $\Phi^{(1)}$ follows from (3.1) and $(5.1)^{2}$. The total force per unit of length on the first beam is then

$$
\underline{K}=\frac{1}{2} \mu_{0} \mu_{r}^{2} R \int_{0}^{2 \pi}\left[\frac{\partial \Phi^{(1)}}{\partial N}\right]^{2} \underline{N} d \phi .
$$

With

$$
\frac{\partial \Phi^{(1)}}{\partial N}=\frac{1}{2} e^{i \phi} \frac{d F^{(1)}}{d z}+\frac{1}{2} e^{-i \phi} \frac{d \bar{F}^{(1)}}{d \bar{z}},
$$

and with use of the results of Section 5, the expression (10.2) can be further evaluated to yield

$$
\begin{aligned}
\underline{K}= & \frac{2 \pi}{\mu_{0}} B_{0}^{2} R \sum_{k=1}^{\infty} k(k+1)\left[\left\{-\left(\delta_{k 1} \zeta_{2}+\zeta_{k} \zeta_{k+1}\right) \cos ^{2} \alpha-\left(\delta_{k 1} \eta_{2}+\eta_{k} \eta_{k+1}\right) \sin ^{2} \alpha\right] \underline{e}_{1}+\right. \\
& \left.+\left\{\left(\zeta_{2}-\eta_{2}\right) \delta_{k 1}-\zeta_{k} \eta_{k+1}+\zeta_{k+1} \eta_{k}\right\} \sin \alpha \cos \alpha \underline{e}_{2}\right]
\end{aligned}
$$

where $\zeta_{k}$ and $\eta_{k}$ are real numbers given by

$$
\zeta_{k}=\sum_{m=1}^{\infty} p_{m} q_{m}^{k}, \quad \eta_{k}=\sum_{m=1}^{\infty}(-1)^{m} p_{m} q_{m}^{k}
$$

and with $p_{m}$ and $q_{m}$ according to (5.6). Note that $\zeta_{k}$ and $\eta_{k}$ depend only on $\delta$ and, hence, not on $\alpha$ (or $\left.\mu_{r}\right)$. Moreover, we conclude that $\underline{K}$ himself does depend on $\alpha$, and that $\left(K, \underline{e}_{2}\right)=0$ for $\alpha=0$ or $\alpha=\pi / 2$ only. We shall further consider these two special cases.

Assuming $\left(\underline{K}, \underline{e}_{1}\right)$ known, we are able to determine the deflection of a rod in the pre-buckled state. We denote this predeflection in the $\underline{e}_{1}$-direction by $U^{(0)}\left(x_{3}\right)$. The deflection $U^{(0)}(l)$ of the end point of a cantilever of length $l$ and bending stiffness $E I$ under a force $K$ per unit of length is given by the well-known formula

$$
U^{(0)}(l)=\frac{K l^{4}}{8 E I} .
$$

In case $\alpha=0$, the force on the first rod acts in the negative $\underline{e}_{1}$-direction, and, hence, the deflection is in 
this direction too. From (10.4) and (10.6) it is deduced that (with $I=\pi R^{4} / 4$ )

$$
U^{(0)}(l)=-\frac{B_{0}^{2} R}{\mu_{0} E}\left(\frac{l}{R}\right)^{4} k_{11},
$$

where

$$
k_{11}=k_{11}(\delta)=2 \zeta_{2}+\sum_{k=1}^{\infty} k(k+1) \zeta_{t} \zeta_{k+1}>0
$$

Using the numerical values given in (8.1) we obtain from (10.7)

$$
U^{(0)}(l)=0.568 k_{11} B_{0}^{2} \quad(m),
$$

where, for instance,

$$
k_{11}= \begin{cases}2.04 \times 10^{-3}, & \text { for } \delta=0.1 \\ 6.53 \times 10^{-3}, & \text { for } \delta=0.4\end{cases}
$$

Taking for $B_{0}$ the critical value at buckling for $\mu_{r}=300$ (i.e. $B_{0}=0,224(T)$ or $B_{0}=4.03 \times 10^{-2}(T)$ for $\delta=0.1$ or $\delta=0.4$, respectively) we find

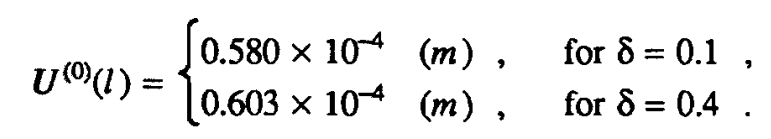

Hence, we conclude that these prebuckling deflections are indeed very small and, moreover, in good (at least in order of magnitude) correspondence with the experimental values which can be read off from Fig. 3 (the point where the deflection line becomes horizontal is a measure for the pre-deflection).

Two final conclusions can be drawn from the above calculations.

1. In case $\underline{\alpha=0}$ both the pre-deflection and the buckling deflection are in the same plane. Hence, if the system is imperfection-sensitive the pre-deflection will lower the actual buckling value. This explains why we experimentally found a somewhat lower buckling value than theoretically predicted. Secondly, because there was a small pre-deflection of the first rod in the negative $\underline{e}_{1-}$ direction, it may be expected that this rod also buckles in this direction (and, thus, the two rods approach each other) and this is just what we observed in our experiments. Again we note that our theory (in which all pre-deflections were neglected) did not indicate any preference for buckling toward each other or away from each other.

2. In case $\underline{\alpha=\pi / 2}$ the plane in which the pre-deflection takes place (i.e. the $\underline{e}_{1}-\underline{e}_{3}$-plane) is perpendicular to the plane of buckling (i.e. the $\underline{e}_{2}-\underline{e}_{3}$-plane). Hence, in this case there is no influence of the pre-deflection on the buckling values and, therefore, a much better correspondence between our theoretical and experimental results is found. Note also that the buckling recorded in the second graph $(\alpha=\pi / 2)$ of Fig. 3 is much sharper than the one in the first graph $(\alpha=0)$. 
In our experiments, conducted for $\alpha=0$ and $\alpha=\pi / 2$ the directions of the buckling deflections and of the basic field $\underline{B}_{0}$ coincide. However, this is only so for these two values of $\alpha$; for all other values of $\alpha \in(0, \pi / 2)$ the buckling displacement $\underline{\mu}$ is not in the same direction as $\underline{B}_{0}$. In order to make this plausible, we assume a symmetrical buckling mode and we consider the deflection of the first rod, i.e.

$$
\underline{u}^{(1)}=u_{1}^{(1)} \underline{e}_{1}+u_{2}^{(1)} \underline{e}_{2} .
$$

Defining $v$ as

$$
v=w^{(1)} e^{-i \alpha}, \quad\left(w^{(1)}=u_{1}^{(1)}+i u_{2}^{(1)}\right),
$$

we see that

$$
v=\left[u_{1}^{(1)} \cos \alpha+u_{2}^{(1)} \sin \alpha\right]+i\left[-u_{1}^{(1)} \sin \alpha+u_{2}^{(1)} \cos \alpha\right] .
$$

Hence, $\operatorname{Re} v$ represents the component of the deflection in the $\underline{B}_{0}$-direction, whereas $\operatorname{Im} v$ stands for the component normal to $\underline{B}_{0}$. After multiplication by $e^{-i \alpha}$, (7.14) yields

$$
\begin{aligned}
& {\left[\left(Q_{1}-Q_{3}-\Omega\right)+\left(Q_{2}-Q_{4}\right) e^{-2 i \alpha}\right] \operatorname{Re} v+} \\
& +\left[\left(Q_{1}-Q_{3}-\Omega\right)-\left(Q_{2}-Q_{4}\right) e^{-2 i \alpha}\right] \operatorname{Im} v=0
\end{aligned}
$$

Without entering into the details, we now state that our calculations reveal that $\left(Q_{1}-Q_{3}\right) \in \mathbb{R}$ and $\left(Q_{2}-Q_{4}\right) \in \mathbb{R}$ for $\alpha=0$ or $\pi / 2$, and for these two values of $\alpha$ only. Furthermore, $\left(Q_{2}-Q_{4}\right)>0$ for $\alpha=0$ and $\left(Q_{2}-Q_{4}\right)<0$ for $\alpha=\pi / 2$. Then (7.16) implies

$$
\Omega=Q_{1}-Q_{3} \pm\left(Q_{2}-Q_{4}\right), \quad \alpha=0, \quad \pi / 2,
$$

where the + sign holds for $\alpha=0$ and the - sign for $\alpha=\pi / 2$. Hence, for both values of $\alpha$ the coefficient of $\operatorname{Re} v$ in (10.15) is zero, implying that $\operatorname{Im} v=0$ and, thus, leading to the conclusion that the buckling deflection is in the same direction as the $\underline{B}_{0}$-field for $\alpha=0$ or $\pi / 2$. We conclude by asserting that for all other values of $\alpha$ these two directions are distinct. This assertion is confirmed by the results of [6], where the same problem, but only for very large values of $\mu_{r}$, is solved by a quite different method based upon a variational principle. In [6] it is shown that the angle between the directions of $\underline{u}$ and $\underline{B}_{0}$ depends on the values of $\alpha$ and $\delta$ (see [6], Fig. 3.2). When we apply our results to the case of very large values of $\mu_{r}$ (e.g. $\mu_{r}=5 \times 10^{4}$ ) we find complete correspondence with the results of [6]. 


\section{References}

[1] Tani, J., Otomo, K.: Interaction of two nearby ferromagnetic panels on the magnetoelastic buckling. Proceedings of the IUTAM-IUPAP Symposium on the Mechanical Behaviour of Electromagnetic Solid Continua, Paris 1983 (Maugin, G.A., ed). Amsterdam, North-Holland.

[2] Hutter, K., Ven, A.A.F. van de: Field matter interactions in thermoelastic solids. (Lecture Notes in Physics, Vol. 88) Berlin: Springer 1978.

[3] Ven, A.A.F. van de: Magnetoelastic buckling of thin plates in a uniform transverse magnetic field. J. of Elasticity $\underline{8}, 297-312$ (1978).

[4] Ven, A.A.F. van de: Magnetoelastic buckling of a beam of elliptic cross-section. Acta Mechanica 51, 119-138 (1984).

[5] Tranter, C.J.: Besselfunctions with some physical applications. London: The English Press Ltd. 1968.

[6] Lieshout, P.H. van, Ven, A.A.F. van de: A variational principle for magneto-elastic buckling. In: Proceedings of the IUTAM-Symposium on the Electromagnetomechanical Interactions in Deformable Solids and Structures, Tokyo, 1986 (Miya, K., ed.) Amsterdam: North-Holland 1987. 